\title{
The Displacement Analysis Investigation for Variable Gradient Parameters Functionally Graded Materials Based on Strip Element Method
}

\author{
J. H. Tian (iD) and L. L. Ma (DD \\ CAE Analysis Room for Engineering Application, School of Mechatronic Engineering, Xian Technological University, \\ Xian 710021, China \\ Correspondence should be addressed to J. H. Tian; 540673737@qq.com
}

Received 26 January 2018; Revised 22 March 2018; Accepted 5 April 2018; Published 24 May 2018

Academic Editor: Francesco Tornabene

Copyright (C) 2018 J. H. Tian and L. L. Ma. This is an open access article distributed under the Creative Commons Attribution License, which permits unrestricted use, distribution, and reproduction in any medium, provided the original work is properly cited.

\begin{abstract}
This paper adopted the strip element method to research the displacement response of functionally graded material structure with variable gradient parameters under mechanical loading. Based on the dynamic equilibrium equation of the system, strip element method theory was established through the principle of virtual work to get the control equations of functionally graded materials plane problem, using the modal superposition method to complete the solution of the equation and obtain the displacement distribution of the FGM plates with variable gradient parameters. The accuracy of the calculation method was verified by comparison with the result of finite element method. The results show that the displacement response of the strip element method is consistent with the finite element method. With the increase of the gradient coefficient, the ceramic content is increased and the material stiffness is also increased; then the displacement response caused by external loading is decreased. With the increase of the gradient coefficient, the influence of the gradient coefficient on the displacement response is decreased gradually.
\end{abstract}

\section{Introduction}

Functionally graded materials (FGM) [1] are a newly developed material in recent years. Their distinguishing feature is that the composition of the material presents a continuous gradient change in the direction of thickness. The feature and function of the material also appear as a gradient change.

A large number of domestic and abroad scholars had done a lot of research on the mechanical problems of functionally graded materials $[2,3]$. Tornabene [4] applied the Generalized Differential Quadrature (GDQ) Method to study four parameters functionally graded and laminated composite shells and panels of revolution. The free vibration of functional gradient materials under mechanical loading and temperature loading was analyzed using Peano-Baker series method by Liu [5]. The dynamic characteristic analytical solution of the quadrilateral and rhombic function gradient plate was obtained by using the beam function group [6]. Tornabene [7] analyzed the dynamic of doubly curved shell structures, and the natural frequencies and mode shapes of several structures are presented and compared to numerical solutions taken from the literature. Viola [8] studied the dynamic behavior of moderately thick functionally graded parabolic panels of revolution and was concerned with the influence of the three parameters of the power-law distribution on their mechanical behavior. Reddy [9] adopted the first-order shear deformation theory to study the structural response of the functional gradient plate structure under the thermomachine coupling. Based on the first-order shear deformation theory (FSDT), Tornabene [10] focused on the dynamic behavior of moderately thick functionally graded parabolic panels and shells of revolution and illustrated the influence of the parameters of the power-law distribution on the mechanical behavior of shell structures. And furthermore the study concerned six types of shell structures that illustrate the influence of the power-law exponent, of the power-law distribution and of the choice of the four parameters on the mechanical behavior of shell structures considered [11]. 
Using the first-order shear deformation theory, Thai [12] had studied the problem of bending and free vibration of functionally graded materials. Liew [13] studied the vibration response of functionally graded material plates under the coupling of mechanical load and electromagnetic load based on the first-order shear deformation theory using the finite element method. Zenkour [14] used first-order shear deformation theory and classical elastic theory to study the structural bending deformation of the sandwich structure under mechanical loading. Ferreira [15] used threeorder shear deformation theory and mesh-less method to study the structure response of functional gradient materials under static load. Reddy [16] studied the deformation of functional gradient rectangular plate based on the threeorder shear deformation theory. Brischetto [17] studied the analytic solution of the structural displacement response of functionally graded material plates under transverse loading. Ray [18] used the finite element method to study the structural response of the functional gradient fiber piezoelectric materials in exponential distribution. Kulikov [19] adopted surface sampling method to solve the problem of threedimensional thermal stress of functional gradient materials.

Due to physical performance parameters of functionally gradient materials with the coordinate changing continuously, the constant coefficient differential equation turns into the variable coefficient differential equation while the theory of elastic body deformation is derived; it is difficult to solve such problem by analytical method. The numerical method is used to solve complex problem while the large computation is in process. The analytical solution is very difficult to obtain, while the precision of the solution will be declined for numerical method for FGM structure, and it also requires a lot of computational memories while using numerical method. In recent years, the researchers have proposed a seminumerical and semianalytic method, that is called the strip element method (SEM) [20], which combined the accuracy of the analytical method with the efficiency of the numerical method.

This study considered the boundary and initially applied strip element method to functionally graded materials structure. Meanwhile this paper firstly considered the displacement response of FGM structure with variable gradient parameters. The paper obtained the displacement response of functionally graded material structure under mechanical loading and further investigated the relationship between the gradient parameters and displacement response of functionally graded material structure. It provides guidance for the application of FGM.

\section{Strip Element Method Theory}

The strip element method is a series of strip elements separated by the solution domain as shown in Figure 1. The displacement function is the continuous function of $x$ direction, and the displacement of the element is obtained through the line displacement interpolation.

Based on the finite element method in $y$ direction, it is assumed that the displacement functions of functionally graded materials are

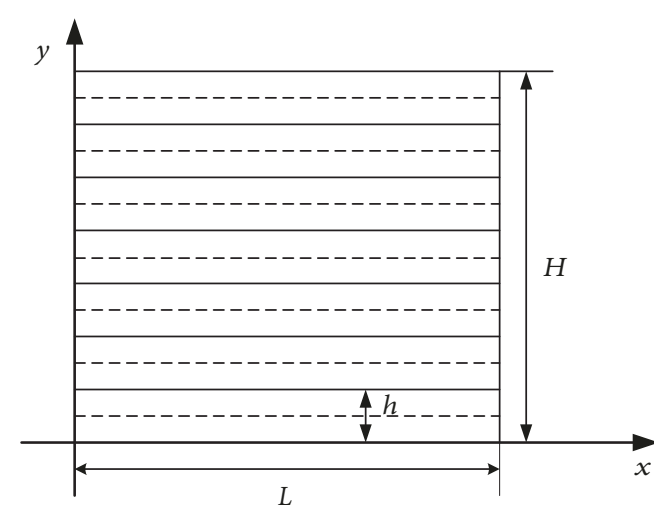

FIGURE 1: Strip element method for solving model.

$$
U=N(y) V(x) \exp (-i \omega t)
$$

where $N(y)$ represents matrix of shape functions for displacement interpolation; $V(x)$ represents continuous function of $x$ direction; $i$ represents a complex unit; $\omega$ represents circle frequency; $t$ represents the computing time.

The shape functions can be derived from the displacement function of a single element. The matrix of shape functions $N(y)[20]$ is

$$
\begin{aligned}
& N(y) \\
& =\left[\left(1-\frac{3 y}{h}+2 \frac{y^{2}}{h^{2}}\right) \mathrm{I} 4\left(\frac{y}{h}-4 \frac{y^{2}}{h^{2}}\right) \mathrm{I}\left(-\frac{y}{h}+2 \frac{y^{2}}{h^{2}}\right) \mathrm{I}\right]
\end{aligned}
$$

where $h$ represents element thickness; I represents the second order identity matrix.

According to Kausel's equation, the dynamic equilibrium equation of the system is

$$
\rho \ddot{U}-L^{T} \sigma=0
$$

where $U$ represents displacement vector; $L$ represent differential operator matrix; $\sigma$ represents stress vector and $\sigma=$ $\left[\begin{array}{lll}\sigma_{x} & \sigma_{y} & \tau_{x y}\end{array}\right]^{T}$; superscript $T$ denotes transpose,

where the expression for $L$ is

$$
L=\left[\begin{array}{cc}
\frac{\partial}{\partial x} & 0 \\
0 & \frac{\partial}{\partial y} \\
\frac{\partial}{\partial y} & \frac{\partial}{\partial x}
\end{array}\right]
$$

It can be seen that the internal displacement of the element is obtained by the interpolation of the nodal displacement from (1). The residual value must be generated in the interpolation calculation, and the residual value is expressed as follows:

$$
W=\rho \ddot{U}-L^{T} \sigma
$$

Since the internal displacement function of the element is interpolated by the nodal displacement, the residual value must exist in the element; then $W \neq 0$. 
According to the principle of virtual work, for a deformed body in equilibrium state, the total virtual work done by the external force is equal to the sum of the work done by the internal force and the work done by the residual value, as follows:

$$
\delta V(x)^{T} F=\delta V(x)^{T} S+\int_{0}^{h} \delta U^{T} W d y
$$

where $\delta V_{t}$ is tiny virtual displacement, $F$ represents external mechanical load, and $S$ represents nodal stress vector.

Given an external load as

$$
F=\bar{F} \exp (-i \omega t)
$$

where $\bar{F}$ represents external load amplitude, the element stress is

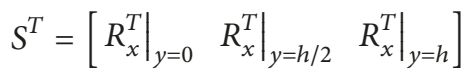

where stress $R_{x}$ is

$$
R_{x}=L_{x}^{T} c L U
$$

where $L_{x}=\left[\begin{array}{ccc}1 & 0 & 0 \\ 0 & 0 & 1\end{array}\right] ; c$ is the elastic constant matrix; $c=$ $\left[\begin{array}{lll}c_{11} & c_{12} & c_{13} \\ c_{21} & c_{22} & c_{32} \\ c_{31} & c_{32} & c_{33}\end{array}\right]$

The displacement function is substituted into the virtual work equation based on the theory of thin plate deformation and we obtain

$$
\begin{aligned}
\bar{F}= & -A_{2} \frac{\partial^{2} V(x)}{\partial x^{2}}+A_{1} \frac{\partial V(x)}{\partial x}+A_{0} V(x) \\
& -\omega^{2} M V(x)
\end{aligned}
$$

where $A_{0}, A_{1}$, and $A_{2}$ represent coefficient matrix. $M$ represents mass matrix.

It can be seen from (10) that the governing equation obtained by element discretization is the ordinary differential equation of $x$, which reduces the difficulty of the solution; it also reflects the idea of $y$ direction discretization.

The coefficient matrix is

$$
\begin{aligned}
& A_{0}=\frac{1}{3 h}\left[\begin{array}{ccc}
7 D_{x x} & -8 D_{x x} & D_{x x} \\
-8 D_{x x} & 16 D_{x x} & -8 D_{x x} \\
D_{x x} & -8 D_{x x} & 7 D_{x x}
\end{array}\right] \\
& A_{1}=\frac{1}{3}\left[\begin{array}{ccc}
3\left(D_{x y}-D_{x y}^{\prime}\right) & -4 D_{x y} & D_{x y} \\
4 D_{x y} & 0 & -4 D_{x y} \\
-D_{x y} & 4 D_{x y} & 3\left(D_{x y}-D_{x y}^{\prime}\right)
\end{array}\right] \\
& A_{2}=\frac{h}{30}\left[\begin{array}{ccc}
4 D_{y y} & 2 D_{y y} & -D_{y y} \\
2 D_{y y} & 16 D_{y y} & 2 D_{y y} \\
-D_{y y} & 2 D_{y y} & 4 D_{y y}
\end{array}\right]
\end{aligned}
$$

where

$$
\begin{aligned}
& D_{x x}=\left[\begin{array}{ll}
c_{11} & c_{13} \\
c_{13} & c_{33}
\end{array}\right] ; \\
& D_{y y}=\left[\begin{array}{ll}
c_{33} & c_{23} \\
c_{23} & c_{22}
\end{array}\right] ; \\
& D_{x y}=\frac{1}{2}\left[\begin{array}{ll}
2 c_{13} & c_{33}+c_{12} \\
c_{33}+c_{12} & 2 c_{23}
\end{array}\right] ; \\
& D_{x y}^{\prime}=\left[\begin{array}{ll}
c_{13} & c_{12} \\
c_{33} & c_{23}
\end{array}\right]
\end{aligned}
$$

The expression for the mass matrix is

$$
M=\int_{0}^{h} \rho N(y)^{T} N(y) d y
$$

Assembling all elements, (10) becomes as

$$
\begin{aligned}
\overline{F_{t}} & =\left[-A_{2 t} \frac{\partial V_{t}(x)}{\partial x^{2}}+A_{1 t} \frac{\partial V_{t}(x)}{\partial x}+A_{0 t} V_{t}(x)\right. \\
& \left.-\omega^{2} M_{t} V_{t}(x)\right]
\end{aligned}
$$

where $A_{0 t}, A_{1 t}$, and $A_{2 t}$ are the coefficients matrix for the element assembly in the solving domain; $M_{t}$ is the mass assembly matrix; $\overline{F_{t}}$ is the external mechanical load; $V_{t}(x)$ is the displacement function in the solving domain.

Assume that the displacement function form is

$$
V_{t}(x)=d_{t} \exp (i k x)
$$

Assume that external mechanical load form is

$$
\overline{F_{t}}=P_{t} \exp (i k x)
$$

Substituting (17) and (18) into (16), we obtain

$$
P_{t}=\left[k^{2} A_{2 t}+i k A_{1 t}+A_{0 t}-\omega^{2} M_{t}\right] d_{t}
$$

$P_{t}$ represents the load acting on the nodal line, while $P_{t}=$ 0 (19) is transformed into a homogeneous equation and the eigenvalue equation for the $\mathrm{k}$ is obtained.

$$
\left[k^{2} A_{2 t}+i k A_{1 t}+A_{0 t}-\omega^{2} M_{t}\right] d_{t}=0
$$

Equation (20) can be written

$$
\left(\left[\begin{array}{cc}
0 & \mathrm{I} \\
\omega^{2} M_{t}-\mathrm{A}_{0 t} & -i A_{1 t}
\end{array}\right]-k\left[\begin{array}{cc}
\mathrm{I} & 0 \\
0 & \mathrm{~A}_{2 t}
\end{array}\right]\right)\left\{\begin{array}{l}
d_{t} \\
k d_{t}
\end{array}\right\}=0
$$

The $2 \mathrm{M}(\mathrm{M}=6 \mathrm{~N}-2(\mathrm{~N}-1)=4 \mathrm{~N}-2)$ eigenvalue is obtained by solving (21), $\mathrm{N}$ is the element number, and the eigenvectors corresponding to the $j$ eigenvalues are represented by $\phi_{j}$

$$
\phi_{j}=\left[\begin{array}{llll}
\phi_{1, j} & \phi_{2, j} & \cdots & \phi_{\mathrm{M}, j}
\end{array}\right]
$$


By means of the modal superposition method, the solution of the equation can be expressed as

$$
V_{t}=\sum_{j=1}^{2 \mathrm{M}} C_{j} \phi_{j} \exp \left(i k_{j} x\right)=G(x) C
$$

where

$$
\begin{aligned}
& G(x)=\left[\begin{array}{ccccc}
\phi_{1,1} X_{1} & \phi_{1,2} X_{2} & & \cdots & \phi_{1,2 M} X_{j} \\
\phi_{2,1} X_{1} & \phi_{2,2} X_{2} & & \cdots & \phi_{2,2 M} X_{j} \\
\vdots & \vdots & \ddots & & \vdots \\
\phi_{M, 1} X_{1} & \phi_{M, 2} X_{2} & & \cdots & \phi_{M, 2 M} X_{j}
\end{array}\right] \\
& X_{j}=\exp \left(i k_{j} x\right)
\end{aligned}
$$

Equation (23) is a fundamental solution of the equation. There are $2 \mathrm{M}$ constants $C$ in this fundamental solution, so it is necessary to determine the unknown coefficients by the boundary conditions.

Since (23) is the fundamental solution in the problem domain, the displacement at any point satisfies (23) and the displacement is satisfied at the right boundary of the solution.

$$
V_{b t}^{R}=\sum_{j=1}^{2 M} C_{j} \phi_{j} \exp \left(i k_{j} x^{R}\right)=G\left(x^{R}\right) C
$$

That can be written as

$$
G\left(x^{R}\right) C=V_{b t}^{R}
$$

In the same way, on the left side

$$
G\left(x^{L}\right) C=V_{b t}^{L}
$$

Equations (27) and (28) are assembled and sorted, and the constant $C$ expression can be obtained

$$
C=G^{-1} V_{b t}=\left[\begin{array}{l}
G\left(x^{R}\right) \\
G\left(x^{L}\right)
\end{array}\right]^{-1} V_{b t}
$$

where $V_{b t}$ is the displacement at the boundary

$$
V_{b t}=\left[\begin{array}{ll}
V_{b t}^{R} & V_{b t}^{L}
\end{array}\right]
$$

By (24), $G\left(x^{R}\right)$ can be expressed as

$$
\begin{gathered}
G\left(x^{R}\right)=\left[\begin{array}{cccc}
\phi_{11} X_{1}^{R} & \phi_{12} X_{2}^{R} & \cdots & \phi_{1 L} X_{j}^{R} \\
\phi_{21} X_{1}^{R} & \phi_{22} X_{2}^{R} & \cdots & \phi_{2 L} X_{j}^{R} \\
\vdots & \vdots & \ddots & \vdots \\
\phi_{M 1} X_{1}^{R} & \phi_{M 2} X_{2}^{R} & \cdots & \phi_{M L} X_{j}^{R}
\end{array}\right] \\
X_{j}^{R}=\exp \left(i k_{j} x^{R}\right)
\end{gathered}
$$

The superscript $R$ represents the right boundary.
Similarly, the left boundary condition has the same form as the displacement matrix at the right boundary. The difference between the two forms is that the $x$ coordinate value at the right boundary is changed into the $x$ coordinate value at the left boundary.

$$
X_{j}^{L}=\exp \left(i k_{j} x^{L}\right)
$$

\section{Application of the Boundary Conditions}

The stress vector of the internal element can be expressed as

$$
R_{x}=D_{x x} \frac{\partial U}{\partial x}+D_{x y}^{\prime} \frac{\partial U}{\partial y}
$$

Substituting (1) into (34), we have

$$
R=R_{1} V(x)+R_{2} \frac{\partial V(x)}{\partial x}
$$

where $R_{1}$ and $R_{2}$ are element coefficient matrices.

$R_{1}$ is

$$
R_{1}=\frac{1}{h}\left[\begin{array}{ccc}
D_{y y} & -4 D_{y y} & 3 D_{y y} \\
-D_{y y} & 0 & D_{y y} \\
-3 D_{y y} & 4 D_{y y} & -D_{y y}
\end{array}\right]
$$

$R_{2}$ is

$$
R_{2}=\left[\begin{array}{ccc}
D_{y x}{ }^{\prime} & 0 & 0 \\
0 & D_{y x}{ }^{\prime} & 0 \\
0 & 0 & D_{y x}{ }^{\prime}
\end{array}\right]
$$

Calculate $R_{1}$ and $R_{2}$ for all elements, and obtain the overall stress vector $R_{t}$ as follows:

$$
R_{t}=R_{1 t} V_{t}(x)+R_{2 t} \frac{\partial V(x)}{\partial x}
$$

where $R_{1 t}$ and $R_{2 t}$ are the assemble matrix of the element coefficient matrix.

The displacement is simplified by using the left and right boundaries

$$
\begin{aligned}
\frac{\partial V(x)}{\partial x} & =\sum_{j=1}^{2 M} i k_{j} \exp \left(i k_{j} x\right) \cdot c_{j}=G^{\prime} \cdot C \\
& =G^{\prime} \cdot G_{d}{ }^{-1} V_{b t}
\end{aligned}
$$

The stress vector of the inner nodal line is equal to the average value of the stress vector of the adjacent left and right elements. We obtain

$$
R_{b t}=K V_{b t}
$$

In (40), $R_{b t}=\left[\begin{array}{ll}R_{b t}^{R} & R_{b t}^{L}\end{array}\right]$ is an external load acting on the left and right boundaries, where the stiffness matrix $K$ is

$$
K=\left[\begin{array}{cc}
R_{1 t} & 0 \\
0 & R_{2 t}
\end{array}\right]+\left[\begin{array}{c}
R_{2 t} G^{R \prime} G_{d}^{-1} \\
R_{2 t} G^{L \prime} G_{d}^{-1}
\end{array}\right]
$$


TABLE 1: Physical properties of $1 \mathrm{Cr}_{18} \mathrm{Ni}_{11} \mathrm{Nb}$ and $\mathrm{Si}_{3} \mathrm{~N}_{4}$ materials.

\begin{tabular}{lcc}
\hline material & Modulus of elasticity/MPa & Poisson's ratio \\
\hline $1 \mathrm{Cr}_{18} \mathrm{Ni}_{11} \mathrm{Nb}$ & $2.38 \times 10^{5}$ & 0.3177 \\
$\mathrm{Si}_{3} \mathrm{~N}_{4}$ & $3.22 \times 10^{5}$ & 0.2400 \\
\hline
\end{tabular}

In (41), $G^{R \prime}$ and $G^{L \prime}$ have the same expressions as $G^{R}$ and $G^{L}$. The difference is that $G^{R}$ and $G^{L}$ in $X_{j}^{R}$ and $X_{j}^{L}$ are replaced by $X_{j}^{R \prime}=i k_{j} \exp \left(i k_{j} x^{R}\right)$ and $X_{j}^{L \prime}=i k_{j} \exp \left(i k_{j} x^{L}\right)$, respectively.

Equation (40) reflects the relationship between the stress boundary condition and displacement; the stress and displacement boundary conditions are transformed into displacement boundary conditions by (40); a constant $C$ can be obtained by substituting the boundary displacement into (29); from (23) and (1) the displacement can be obtained in the solving problem.

\section{Establishment of Gradient Parameter Model}

The functional gradient materials studied in this paper and the model is composite by metal and ceramic materials. The metal material is $1 \mathrm{Cr}_{18} \mathrm{Ni}_{11} \mathrm{Nb}$ and the ceramic material is $\mathrm{Si}_{3} \mathrm{~N}_{4}$. The physical properties of the two materials are shown in Table 1.

Because functionally graded materials are continuously changed by different materials according to the design requirements, the physical performance parameters of the materials are expressed as a function of the volume fraction of the material, the physical properties of the materials, and the content of components; it denotes

$$
\begin{gathered}
E(y)=\left(E_{\mathrm{C}}-E_{\mathrm{M}}\right) Q_{\mathrm{C}}+E_{\mathrm{M}} \\
\nu(y)=\left(v_{\mathrm{C}}-v_{\mathrm{M}}\right) Q_{\mathrm{C}}+\nu_{\mathrm{M}} \\
\rho(y)=\left(\rho_{\mathrm{C}}-\rho_{\mathrm{M}}\right) Q_{\mathrm{C}}+\rho_{\mathrm{M}}
\end{gathered}
$$

where $E(y), v(y)$, and $\rho(y)$ are the elastic modulus, Poisson's ratio, and density of FGM plates, respectively. $E_{\mathrm{C}}, v_{\mathrm{C}}$, and $\rho_{\mathrm{C}}$ are the elastic modulus, Poisson's ratio, and density of ceramic materials, respectively. $E_{\mathrm{M}}, \nu_{\mathrm{M}}$, and $\rho_{\mathrm{M}}$ are the elastic modulus, Poisson's ratio, and density of metal materials, respectively. $Q_{C}$ is the volume fraction of the ceramic.

To ensure the continuity of the material, the sum of the metal volume fraction and the ceramic volume fraction at any location of the material is 1 .

$$
Q_{C}+Q_{M}=1
$$

where $Q_{M}$ is the volume fraction of metals.

The volume fraction change function of the functionally graded metal is

$$
Q_{\mathrm{M}}=\left(1-a\left(1-\frac{y}{H}\right)+b\left(1-\frac{y}{H}\right)^{c}\right)^{p}
$$

where $y$ is the vertical position and $0 \leq y \leq H$; $H$ is FGM plate thickness; $p \geq 0$ is the gradient parameters.

By (44), it can be seen that when $p=0, Q_{M}=1$ and $Q_{C}=0$. Substituting that into (42) we have $E(y)=E_{M}$,

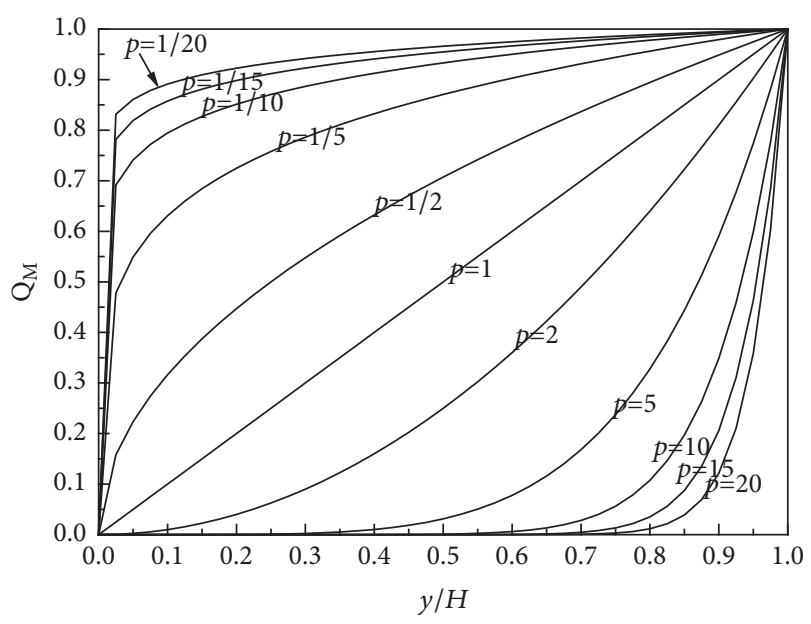

FIGURE 2: Law of change of volume fraction of metal with thickness under different gradient parameters at $a=1$ and $b=0$.

$\nu(y)=\nu_{\mathrm{M}}$, and $\rho(y)=\rho_{\mathrm{M}}$ and functionally graded material degenerates into pure metal homogeneous material. When $p$ tends to infinity, $Q_{C}$ tends to 1 and (42) can be obtained $E(y)=E_{\mathrm{C}}, \nu(y)=\nu_{\mathrm{C}}$, and $\rho(y)=\rho_{\mathrm{C}}$ and the material is reduced to a pure ceramic homogeneous material.

When $a=1$ and $b=0$ are functionally graded materials, the volume fraction of metals varies as shown in Figure 2. Figure 2 can be shown: when $y=0$, metal volume fraction is 0 and this position is pure ceramic material; when $y=H$, metal volume fraction is equal to 1 and this position is the pure metal homogeneous material. So when $a=1$ and $b=0$, the transition form of materials is a continuous transition of ceramic to metal. When the gradient parameter $p=1$, the change of the volume fraction of the functionally graded material is continuous linear change. When the gradient parameter is lesser than 1 , the change of the metal volume fraction decreases. When the gradient parameter is greater than 1 , the metal volume fraction of the functional gradient material is slower in the early and then the volume fraction is accelerated with the thickness increased.

\section{Computational Model}

5.1. Validation of Strip Element Method. Based on the finite element (FEM) theory, we used ANSYS to verify the results of SEM solution. The model of Figure 3 is solved by using the strip element method and finite element method. In Figure $3, L=100 \mathrm{~mm}, H=100 \mathrm{~mm}$, the lower boundary is fixed, $q=100 \mathrm{~N} / \mathrm{mm}$, and uniform load is applied on the upper boundary. The solution domain is divided into 20 elements and the material properties in each element are metal material. The metallic material property is shown in Table 1. The displacement of $x$-direction and $y$-direction at $y=10 \mathrm{~mm}, y=50 \mathrm{~mm}$, and $y=90 \mathrm{~mm}$ is shown in Figures 4 and 5 by strip element method and finite element method (FEM).

From Figures 4 and 5 we can see that the displacement obtained by strip element method and finite element method in $x$-direction and $y$-direction at different position is almost 


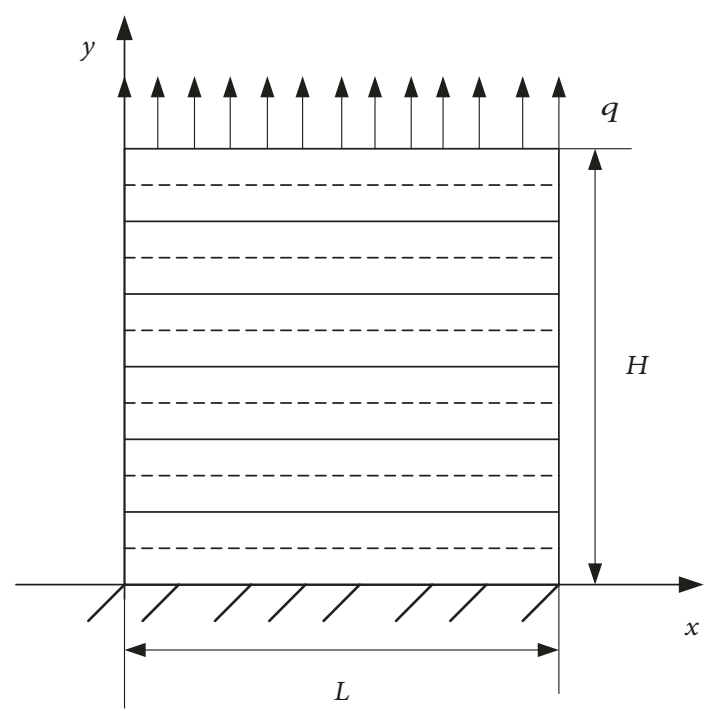

Figure 3: Physical model of plane problem.

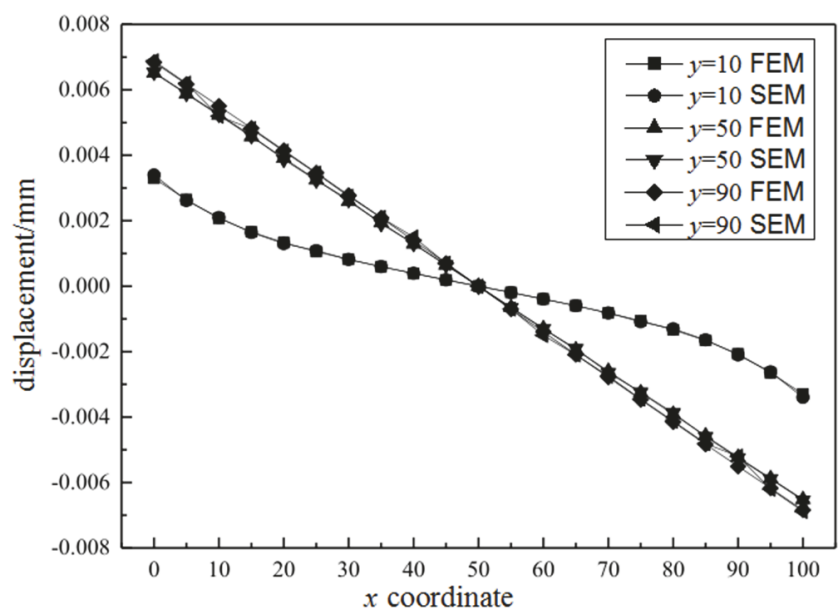

FIGURE 4: The displacement in $x$-direction in different position by FEM and SEM.

the same. From $y=10 \mathrm{~mm}$ and $y=50 \mathrm{~mm}$ to $y=90 \mathrm{~mm}$, the displacement in $y$-direction is increased. The accuracy of the strip element method is verified.

5.2. The Influence of Element Number for the Results. In the calculation process, because of the discrete of the solving domain, the size of the element has an influence on the accuracy of the displacement solution. Figures 6 and 7 are the $x$-direction displacement and the $y$-direction displacement at $y=10 \mathrm{~mm}$ using 10 elements, 20 elements, and 50 elements, respectively. It can be known that displacement results have small difference when the element numbers are 10 and 20, while when the element numbers are 20 and 50, the result difference is not obvious, so in this calculation the element number is set to 20 for ensuring that precision and calculation speed.

5.3. Structural Response Analysis of Functionally Graded Materials. The displacements with different gradient parameters

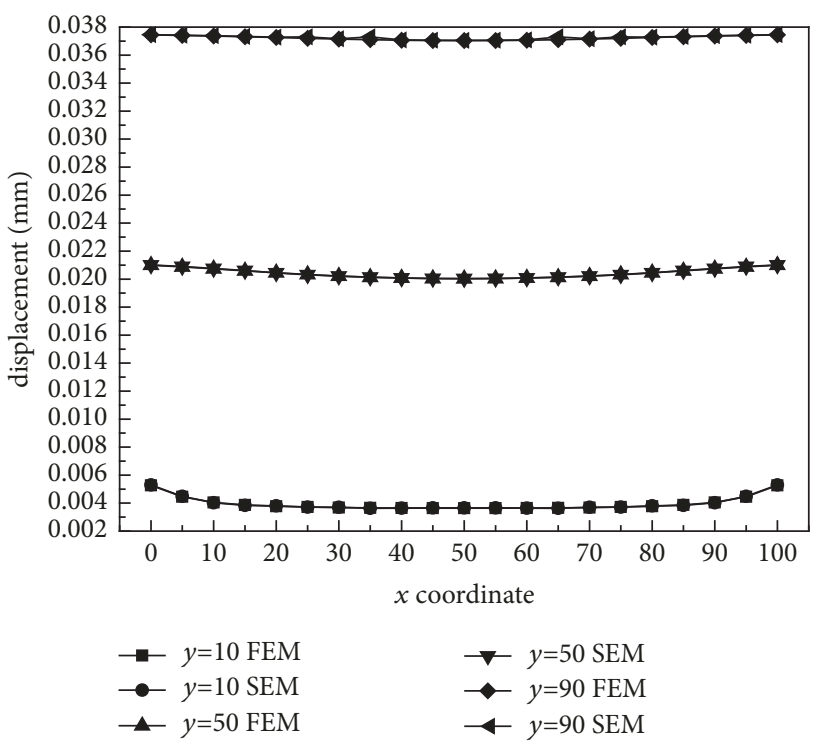

FIGURE 5: The displacement in $y$-direction in different position by FEM and SEM.

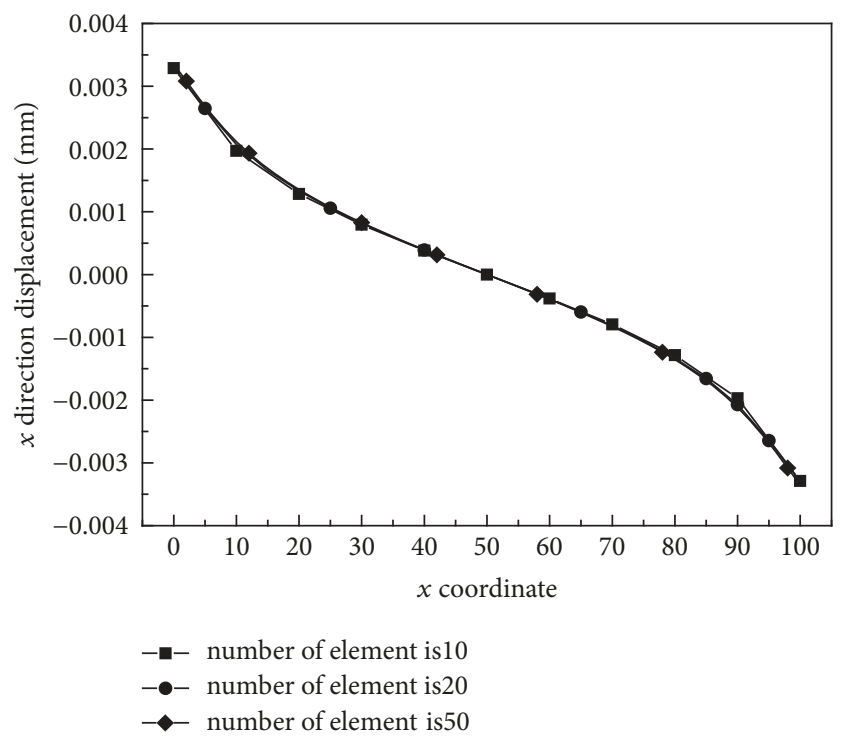

FIGURE 6: Calculation results of $x$ direction displacement at $y=$ $10 \mathrm{~mm}$.

are shown in Figure 8 at $y=10 \mathrm{~mm}$ in the $x$ direction. It can be seen from Figure 8, with the increase of gradient coefficient, that the displacement response decreases, and this is due to the fact that metal volume fraction decreases in the functionally graded materials and ceramic volume fraction increases, causing the structure stiffness to increase. In addition, the displacement in the $x$ direction at $y=10 \mathrm{~mm}$ has nonlinear characteristics.

The displacements with different gradient parameters are shown in Figure 9 at $y=10 \mathrm{~mm}$ in the $y$ direction. It can be seen from Figure 9 that the displacement in $y$ direction at $y=10 \mathrm{~mm}$ with the increase of $x$ coordinate has the characteristics of first increasing, then decreasing, and then increasing. As the gradient parameter increases, the 


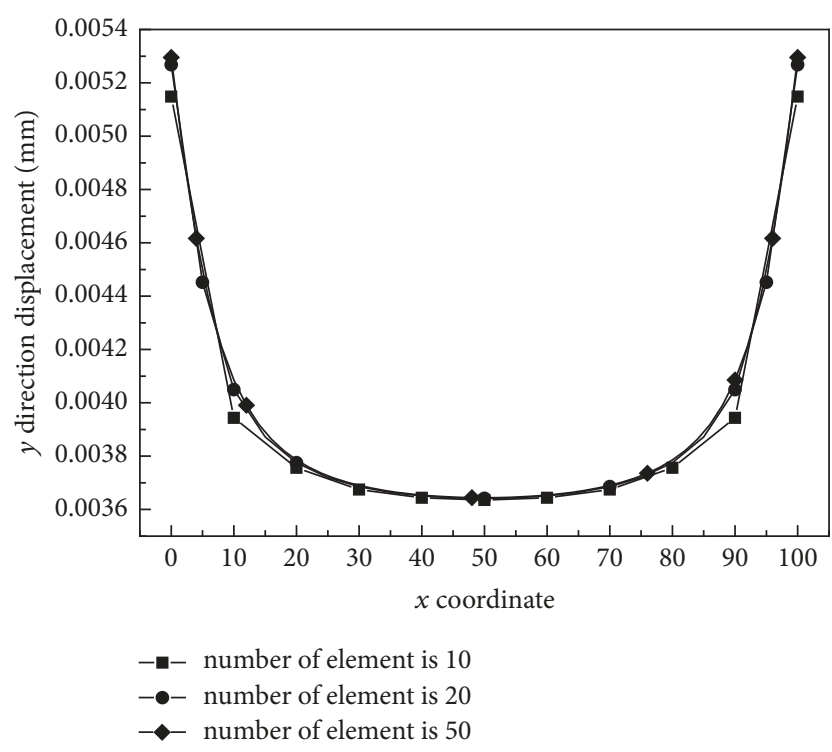

Figure 7: Calculation results of $y$ direction displacement at $y=$ $10 \mathrm{~mm}$.

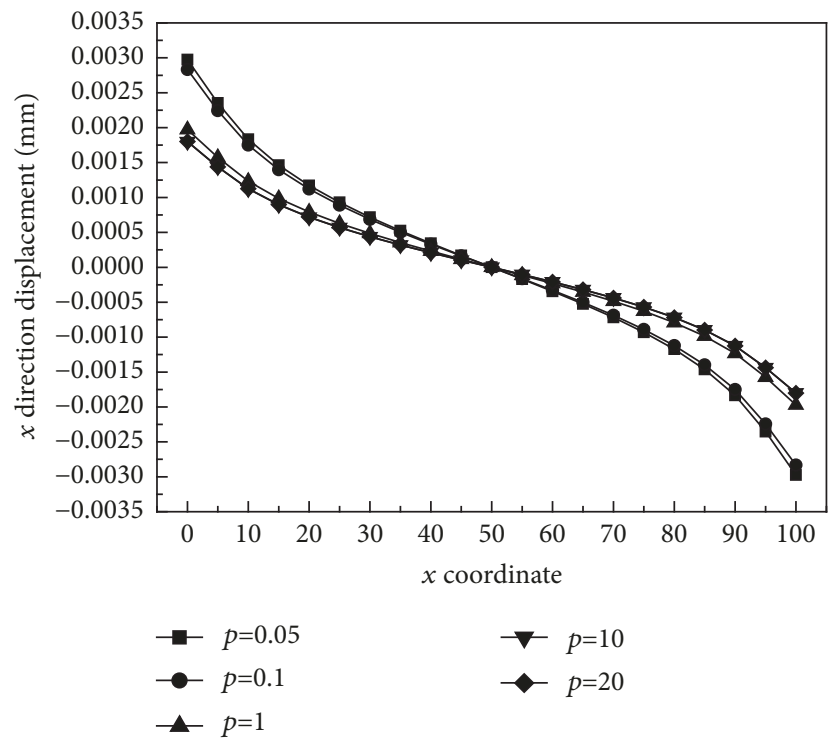

FIGURE 8: $x$ direction displacement of different gradient parameters at $y=10 \mathrm{~mm}$.

displacement of the same position decreases. As the gradient coefficient increases, the influence of the gradient coefficient for the displacement response decreases gradually.

$x$ direction of displacement is shown in Figures 10 and 11 at $y=50 \mathrm{~mm}$ and $y=90 \mathrm{~mm}$. They have the same law at $y=50 \mathrm{~mm}$ and $y=90 \mathrm{~mm}$. The displacement absolute value of $x$ direction with respect to $x=50 \mathrm{~mm}$ is symmetrical and, with the increase of gradient coefficient, $x$ direction displacement is reduced; this is due to the increase of the gradient coefficient; the ceramics content of the material keeps increasing causing the structure stiffness to increase.

$y$ direction of displacement is shown in Figures 12 and 13 at $y=50 \mathrm{~mm}$ and $y=90 \mathrm{~mm}$. It can be seen that the displacement in $y$ direction has class parabolic distribution,

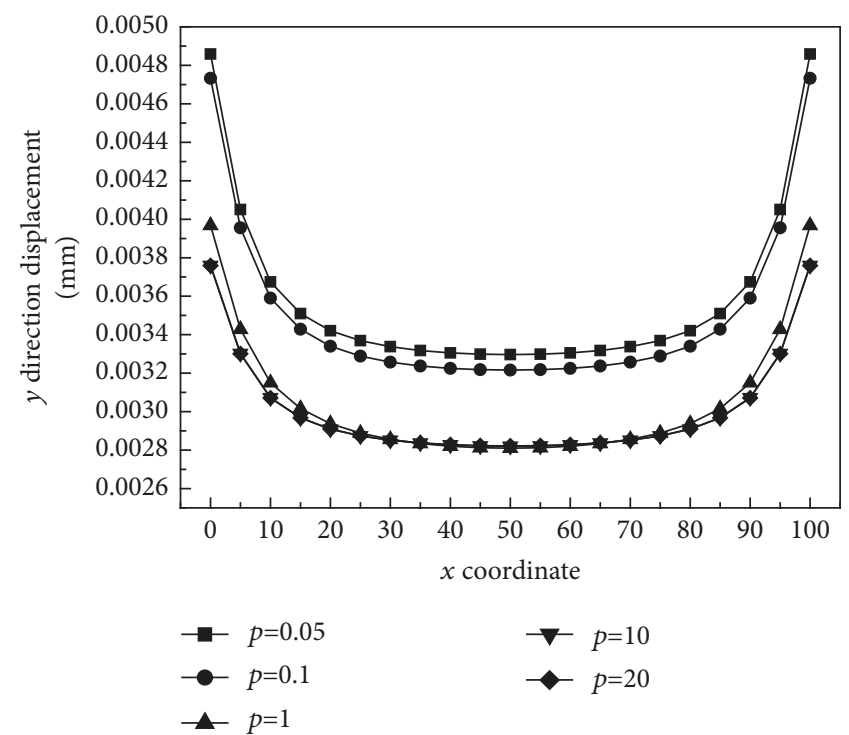

FIGURE 9: $y$ directional displacement of different gradient parameters at $y=10 \mathrm{~mm}$.

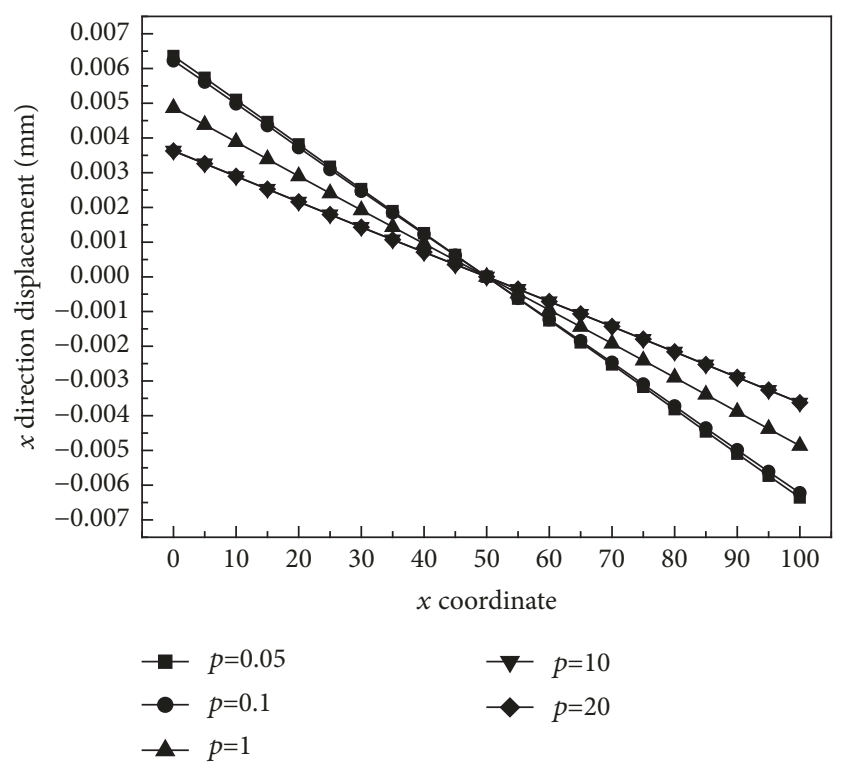

FIGURE 10: $x$ directional displacement of different gradient parameters at $y=50 \mathrm{~mm}$.

and as the change of spatial coordinates, the curve is approximatively changed to straight with the increase of gradient coefficient, structural stiffness is gradually increased and the displacement response under external loading is reduced.

\section{Conclusion}

This paper presents the variable gradient parameters model of the functional gradient materials. The displacement response of considering different gradient parameters model is analyzed in mechanics loading using the strip element method; there are some useful results. 


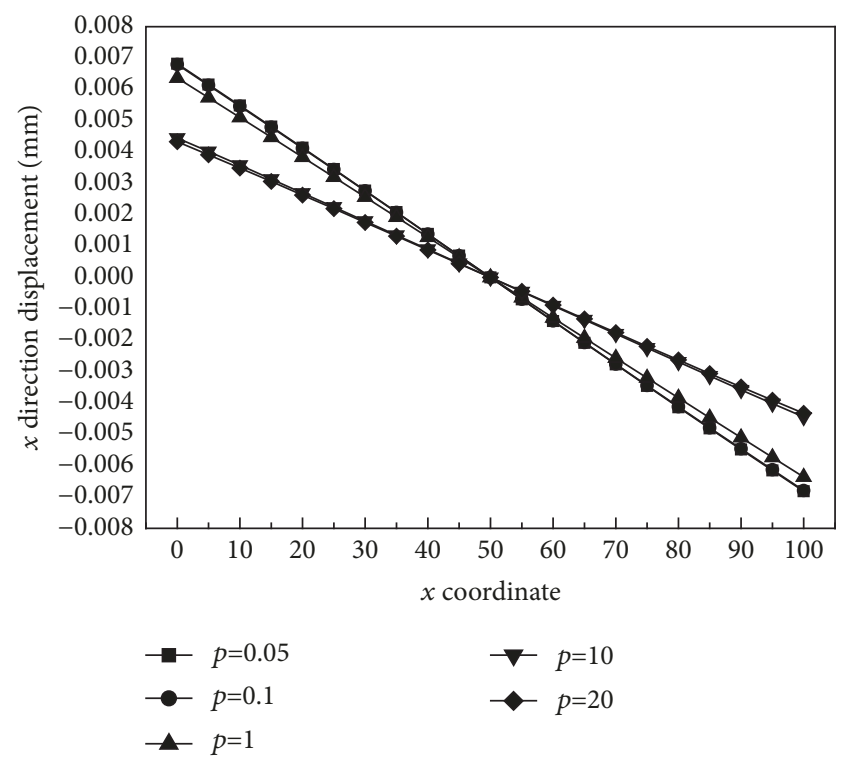

FIGURE 11: $x$ direction displacement for different gradient parameters at $y=90 \mathrm{~mm}$.

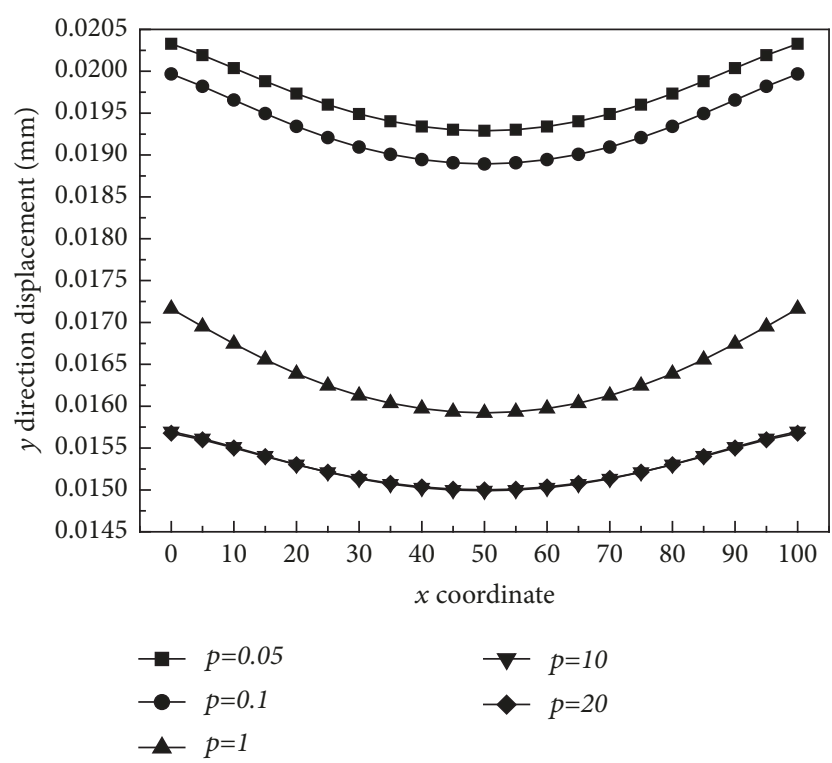

FIGURE 12: $y$ direction displacement of different gradient parameters at $y=50 \mathrm{~mm}$.

(1) The displacement response result is almost the same by using finite element method and strip element method, so as to verify the validation of the strip element method.

(2) With the ceramic volume fraction increasing, the metal volume fraction decreases in the functionally graded materials, the structure stiffness increases, and displacement response decreases.

(3) As the gradient coefficient increases, the influence of the gradient coefficient on the structure displacement response decreases gradually.

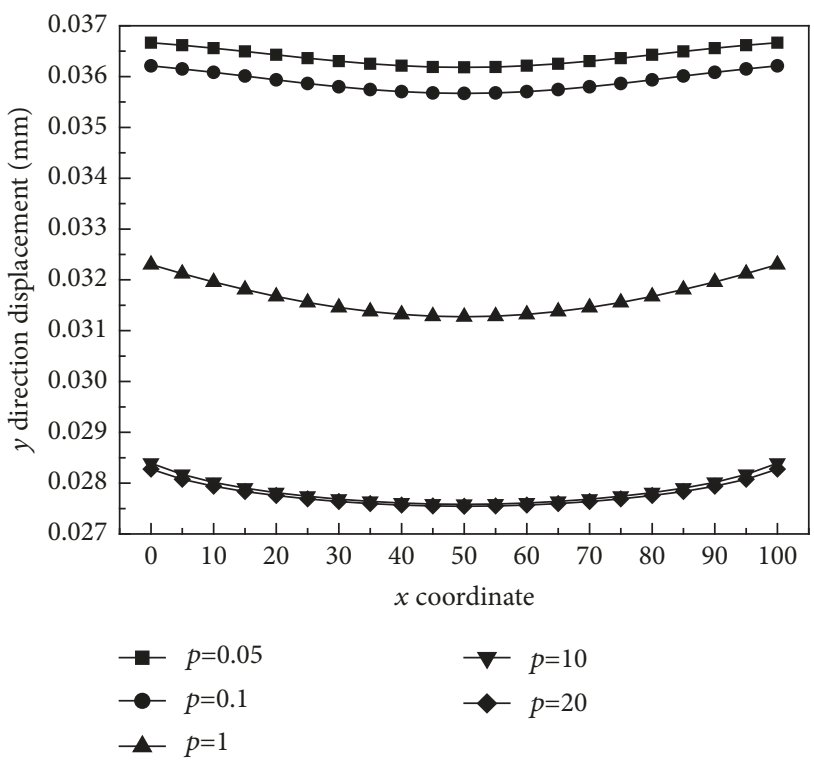

FIGURE 13: $y$ direction displacement for different gradient parameters at $y=90 \mathrm{~mm}$.

\section{Data Availability}

The data used to support the findings of this study are available from the corresponding author upon request.

\section{Disclosure}

This work was presented at "the 8th International Conference on Computational Methods (ICCM2017)" for communication.

\section{Conflicts of Interest}

The authors declare that they have no conflicts of interest.

\section{Acknowledgments}

The present paper was financially supported by the National Natural Science Foundation of China (no. 11302159), Young Talent Fund of University Association for Science and Technology in Shaanxi (no. 20170519), and the Principal Foundation of Xi'an Technological University (no. XAGDXJJ16009).

\section{References}

[1] M. Koizumi, "FGM activities in Japan," Composites Part B: Engineering, vol. 28, no. 1-2, pp. 1-4, 1997.

[2] Z. Zhen, W. Linzhi, and C. Weiqiu, "Progress in the Study on Mechanics Problems of Functionally Graded Materials and Structures," Advances in Mechanics, vol. 40, no. 5, pp. 528-541, 2010.

[3] Z. Cao, "Unified expression of natural frequency solutions for functionally graded composite rectangular plates under various boundary conditions," Fuhe Cailiao Xuebao/Acta Materiae Compositae Sinica, vol. 22, no. 5, pp. 172-177, 2005. 
[4] F. Tornabene and E. Viola, "Static analysis of functionally graded doubly-curved shells and panels of revolution," Meccanica, vol. 48, no. 4, pp. 901-930, 2013.

[5] W. X. Liu, "Three-dimensional thermoelastic analysis on functionally graded plates with arbitrary graded distribution functions," Tongji Daxue Xuebao. Ziran Kexue Ban. Journal of Tongji University. Natural Science, vol. 40, no. 2, pp. 205-210, 2012.

[6] C. A. O. Zhiyuan, "Analytical Solution of Dynamic Character for Functionally Graded Material Parallelogrammic Plates with Various Boundary Conditions," Chinese Quarterly of Mechanics, vol. 27, no. 2, pp. 255-261, 2006.

[7] F. Tornabene, N. Fantuzzi, and M. Bacciocchi, "Free vibrations of free-form doubly-curved shells made of functionally graded materials using higher-order equivalent single layer theories," Composites Part B: Engineering, vol. 67, pp. 490-509, 2014.

[8] E. Viola and F. Tornabene, "Free vibrations of three parameter functionally graded parabolic panels of revolution," Mechanics Research Communications, vol. 36, no. 5, pp. 587-594, 2009.

[9] J. N. Reddy, "Analysis of functionally graded plates," International Journal for Numerical Methods in Engineering, vol. 47, no. 1-3, pp. 663-684, 2000.

[10] F. Tornabene and E. Viola, "Free vibrations of four-parameter functionally graded parabolic panels and shells of revolution," European Journal of Mechanics - A/Solids, vol. 28, no. 5, pp. 9911013, 2009.

[11] F. Tornabene, "Free vibration analysis of functionally graded conical, cylindrical shell and annular plate structures with a four-parameter power-law distribution," Computer Methods Applied Mechanics and Engineering, vol. 198, no. 37-40, pp. 29112935, 2009.

[12] H.-T. Thai and D.-H. Choi, "A simple first-order shear deformation theory for the bending and free vibration analysis of functionally graded plates," Composite Structures, vol. 101, pp. 332-340, 2013.

[13] K. M. Liew, X. Q. He, and S. Kitipornchai, "Finite element method for the feedback control of FGM shells in the frequency domain via piezoelectric sensors and actuators," Computer Methods Applied Mechanics and Engineering, vol. 193, no. 3-5, pp. 257-273, 2004.

[14] A. M. Zenkour, "A comprehensive analysis of functionally graded sandwich plates: part 1-deflection and stresses," International Journal of Solids and Structures, vol. 42, no. 18-19, pp. 5224-5242, 2005.

[15] A. J. M. Ferreira, R. C. Batra, C. M. C. Roque, L. F. Qian, and P. A. L. S. Martins, "Static analysis of functionally graded plates using third-order shear deformation theory and a meshless method," Composite Structures, vol. 69, no. 4, pp. 449-457, 2005.

[16] J. N. Reddy and C. D. Chin, "Thermomechanical analysis of functionally graded cylinders and plates," Journal of Thermal Stresses, vol. 21, no. 6, pp. 593-626, 1998.

[17] S. Brischetto and E. Carrera, "Advanced mixed theories for bending analysis of functionally graded plates," Computers \& Structures, vol. 88, no. 23-24, pp. 1474-1483, 2010.

[18] M. C. Ray and H. M. Sachade, "Finite element analysis of smart functionally graded plates," International Journal of Solids and Structures, vol. 43, no. 18-19, pp. 5468-5484, 2006.

[19] G. M. Kulikov and S. V. Plotnikova, "A sampling surfaces method and its implementation for 3D thermal stress analysis of functionally graded plates," Composite Structures, vol. 120, pp. 315-325, 2015.
[20] G. R. Liu and J. D. Achenbach, "A strip element method for stress analysis of anisotropic linearly elastic solids," American Society of Mechanical Engineers. Transactions of the ASME. Journal of Applied Mechanics, vol. 61, no. 2, pp. 270-277, 1994. 


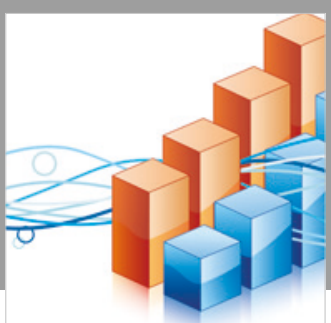

Advances in

Operations Research

\section{-n-m}
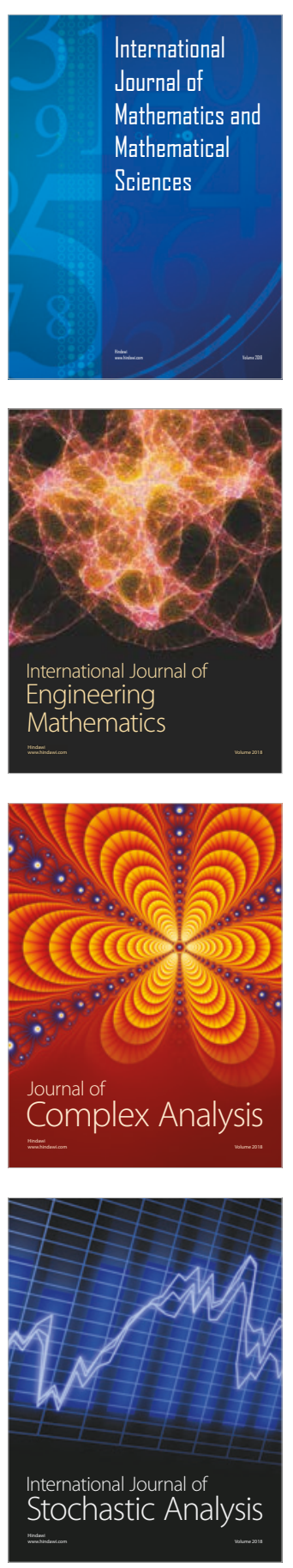
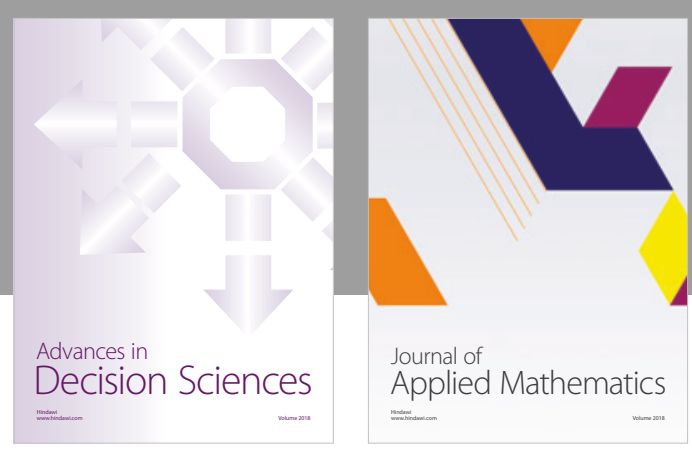

Journal of

Applied Mathematics
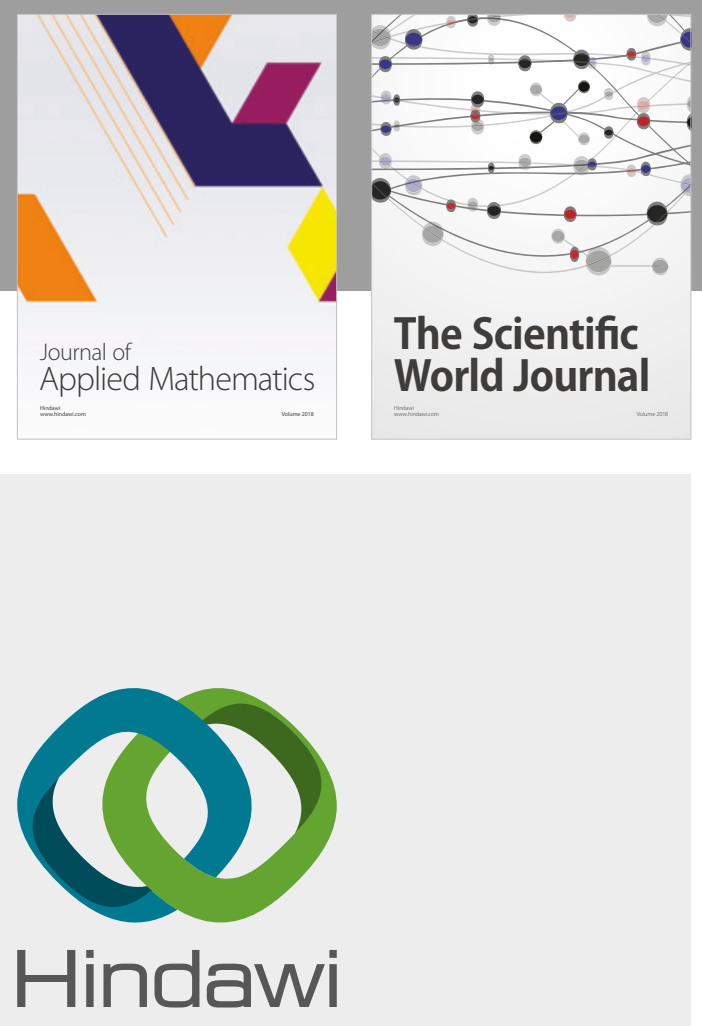

Submit your manuscripts at

www.hindawi.com

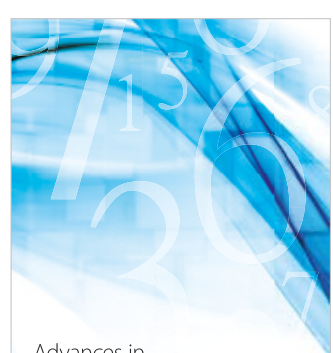

Advances in
Numerical Analysis
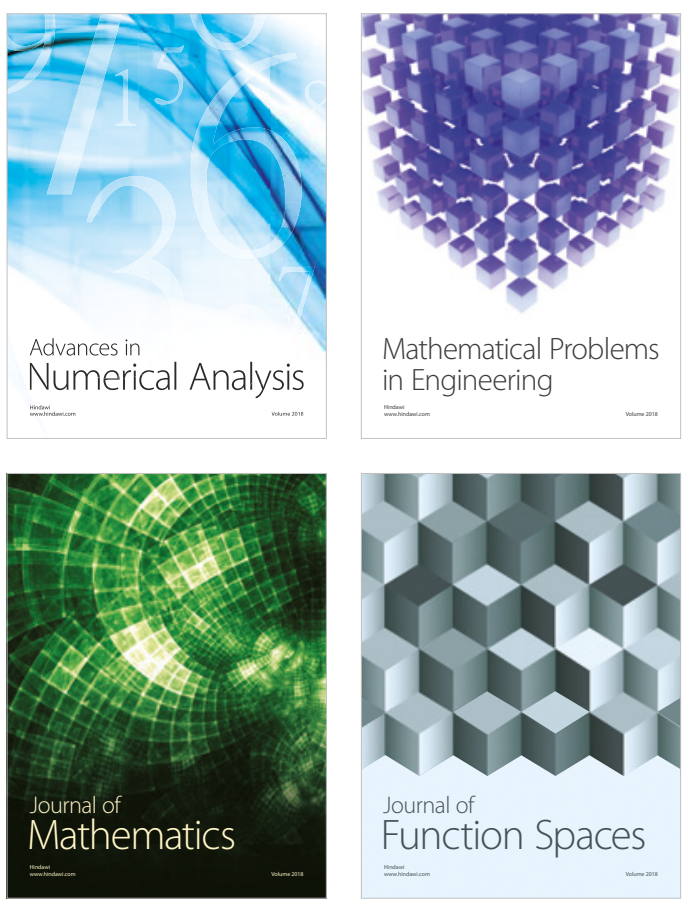

Mathematical Problems in Engineering

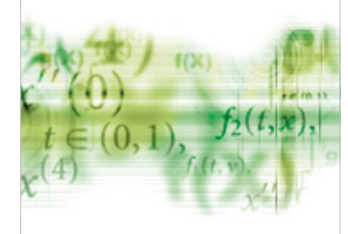

International Journal of

Differential Equations

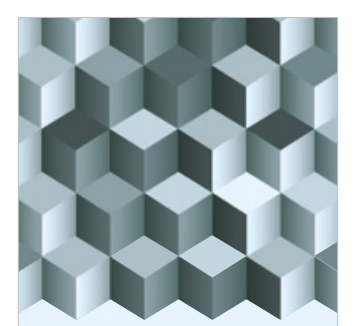

Journal of

Function Spaces

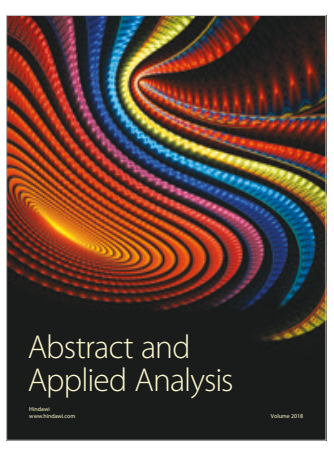

The Scientific

World Journal

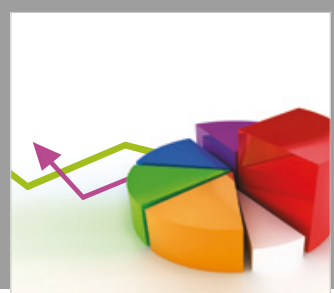

Journal of

Probability and Statistics
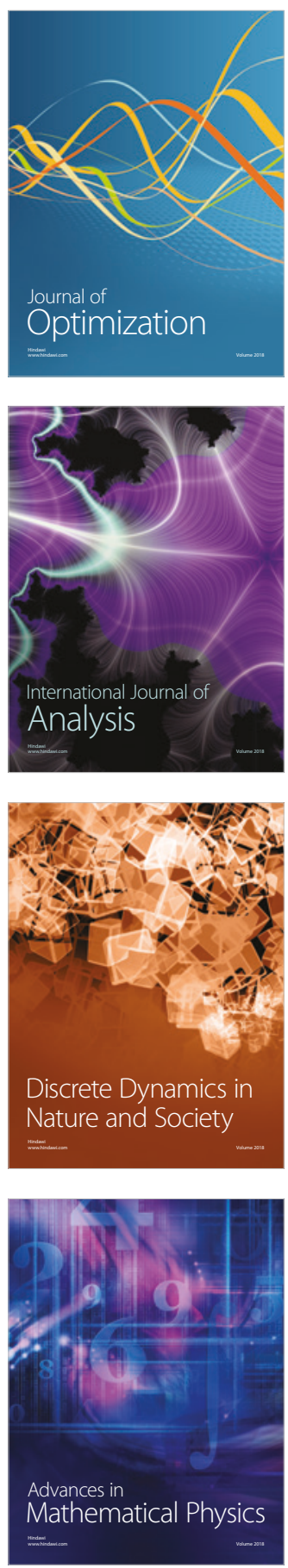